\title{
A-04
}

\section{ESTIMACIÓN DE LA EVAPORACIÓN/TRANSPIRACIÓN EN UN CULTIVO DE VIÑA MEDIANTE RADIOMETRÍA TÉRMICA}

\author{
Sánchez, J.M. ${ }^{(1)}$, López-Urrea, . $^{(2)}$, Caselles, $V^{(3)}$, Montoro, $A .{ }^{(4)}$, Martínez, $L .^{(5)}$, Doña,.$^{(6)}$ \\ 1 Profesor, UCLM, Dpto. Física Aplicada, EPC, Campus Universitario, 16071 Cuenca, \\ juanmanuel.sanchez@uclm.es \\ ${ }^{2}$ Director, ITAP-FUNDESCAM, Polígono Campollano, Avda. 2a-42 B, 02007 Albacete. \\ rlu.itap@dipualba.es \\ ${ }^{3}$ Catedrático, UV, Dpto. Física de la Tierra y Termodinámica, C/Dr. Moliner 50, 46100 \\ Burjassot, Vicente.caselles@uv.es \\ ${ }^{4}$ Investigadora, ITAP-FUNDESCAM, Polígono Campollano, Avda. 2a-42 B, 02007 Albacete. \\ meli.itap@dipualba.es
}

5 Técnica, ITAP-FUNDESCAM, Polígono Campollano, Avda. 2a -42 B, 02007 Albacete. Imm.itap@dipualba.es

${ }^{6}$ Doctoranda, UV, Dpto. Física de la Tierra y Termodinámica, C/Dr. Moliner 50, 46100 Burjassot, carolina.dona@uv.es

\section{Resumen}

La estimación precisa de la evapotranspiración de cultivo $\left(\mathrm{ET}_{\mathrm{c}}\right)$, así como su partición en las componentes evaporativa $(E)$ y transpirativa $(T)$, resultan fundamentales para mejorar la eficacia en la gestión del riego de cultivos en hilera en regiones áridas y semiáridas. El objetivo de este trabajo es contribuir a un mejor entendimiento de la partición E/T sobre viña llevando a cabo un balance de energía por separado para las componentes de suelo y de vegetación. En este trabajo se presentan los resultados de un experimento llevado a cabo en la finca de Las Tiesas, Albacete, de junio a octubre de 2013. Se instalaron un conjunto de radiómetros de infrarrojo térmico, apuntando directamente a las plantas y al suelo entre hileras. Se tomaron medidas de las cuatro componentes de la radiación neta, del flujo de calor en el suelo, además de variables meteorológicas y parámetros biofísicos. Todas las medidas se almacenaron en intervalos de 15 minutos, y se promediaron después a escala horaria y diaria. El valor medio observado de $\mathrm{ET}_{\mathrm{c}}$ fue de 3,1 $\mathrm{mm}$ día $^{-1}$. La evaporación se estimó en torno al $30 \%$. Los resultados presentados en este trabajo ofrecen una primera impresión de la partición E/T, y muestran el potencial de la caracterización térmica de la viña con este fin.

\section{Abstract}

An accurate estimation of crop evapotranspiration $\left(E T_{c}\right)$, and its partition into both components, soil evaporation (E) and canopy transpiration (T), is known to be critical for a more effective irrigation scheduling of row-crops in arid and semi-arid environments. Vineyards are the best example in Mediterranean countries. The aim of this work is to improve our understanding of the E/T partition in vineyards by establishing a separate energy balance for soil and canopy components. An experiment was conducted in Las Tiesas experimental farm, Albacete (Spain), from June to October of 2013. A set of thermal-infrared radiometers were assembled pointing directly to the plants and the soil between rows. Measurements of the four components of the net radiation over the canopy and soil heat fluxes, as well as meteorological variables and biophysical parameters were all collected and stored every 15-min. Hourly and daily averages were then computed and analyzed. An average daily $\mathrm{ET}_{\mathrm{c}}$ value of $3.1 \mathrm{~mm}^{\text {day }}{ }^{-1}$ was observed in both sites. Interrow soil evaporation 
reached as much as $30 \%$ of the total $\mathrm{ET}_{\mathrm{c}}$. These results provide a first insight into the partition $\mathrm{E} / \mathrm{T}$ and show the potential of the vine thermal characterization with this aim.

\section{1- Introducción}

Estimar de forma precisa la evapotranspiración real de cultivo $\left(E T_{c}\right)$ resulta fundamental para mejorar la gestión de los recursos hídricos, así como para lograr una programación del riego más eficiente. Mejorar nuestra comprensión de la partición de $\mathrm{ET}_{\mathrm{c}}$ en sus componentes evaporativa (E) y transpirativa $(T)$ contribuiría en gran medida, especialmente en cultivos no extensivos y en regiones áridas y semiáridas en las que la $E$ juega un papel importante. La viña es un claro ejemplo de cultivo en que se aprecian estas condiciones.

Recientemente se han aplicado y validado diferentes técnicas y métodos para estimar la $\mathrm{ET}_{\mathrm{c}}$ en viña. Shapland et al. (2012) obtuvieron la $\mathrm{ET}_{\mathrm{c}}$ en una viña utilizando la técnica surface renewal. Zhang et al. (2010) estudiaron la variación de $\mathrm{ET}_{\mathrm{c}}$ en una región árida del noroeste de China empleando el método de balance de energía Bowen Ratio. Ortega-Farias et al. (2009) evaluaron el modelo de Shutteworth y Wallace en una viña de la variedad Merlot en Chile. Miglietta et al. (2009) aplicaron una metodología basada en una formulación simplificada del flujo de calor sensible utilizando medidas aéreas en el suroeste de Francia. González-Dugo et al. (2010) comprobaron el funcionamiento del modelo de balance de energía de dos fuentes (TSEB) en una viña de riego por goteo empleando medidas de temperaturas radiométricas en la zona central de España. En la misma viña, Campos et al. (2009) estimaron $\mathrm{ET}_{\mathrm{c}}$ haciendo uso de la técnica del coeficiente de cultivo dual. En todos estos trabajos se obtuvieron resultados satisfactorios en términos de $\mathrm{ET}_{\mathrm{c}}$ total. Además, los modelos TSEB permiten la separación de $\mathrm{E}$ y $\mathrm{T}$ mediante el establecimiento de un balance por separado de las componentes de suelo y de vegetación (Colaizzi et al. 2012; Kustas et al. 2012); sin embargo, la partición entre E y T no es sencilla de validar, y muy pocos trabajos se han ocupado de esta separación de componentes. Algunos esfuerzos se han centrado en medir por separado ambas componentes empleando, por ejemplo, medidas de flujo de savia, instalando micro-lisímetros, o incluso sistemas eddycovariance bajo la vegetación (Wilson et al. 2001; Ham et al. 1990; Yunusa et al. 2004; Colaizzi et al. 2012; Evett et al. 2012). Las medidas de flujo de savia proporcionan una estimación de la transpiración de la planta, sin embargo la representatividad de estas medidas es bastante cuestionable. La instalación de varios micro-lisímetros para medir la evaporación del suelo es una buena solución, aunque bastante costosa. Tampoco se libra de ciertos problemas el uso de sistemas eddy-covariance cerca del nivel del suelo.

En este trabajo continuamos con el estudio iniciado en Sánchez et al. (2015). En dicho trabajo se comprobó el potencial del modelo STSEB (Sánchez et al. 2008, 2014) para estimar de manera aislada la transpiración en una viña de variedad Tempranillo en la zona centro de España. Siguiendo el procedimiento definido en Sánchez et al. (2014), se tomaron medidas de las temperaturas del suelo y de la vegetación, además de variables meteorológicas. Todos estos datos se registraron y almacenaron en intervalos de 15 minutos. En este trabajo se presentan los resultados de un experimento llevado a cabo durante la campaña de 2013 en una viña situada en la finca de Las Tiesas, Albacete.

\section{2- Materiales y métodos}

La nueva experiencia se llevó a cabo entre julio y octubre de 2013 en la finca experimental de "Las Tiesas" ( $2^{\circ} 5^{\prime} \mathrm{W}, 39^{\circ} 3^{\prime} \mathrm{N}, 695 \mathrm{~m}$ sobre el nivel del mar), Albacete (Figura 1). El clima es semiárido, continental con $320 \mathrm{~mm}$ de precipitación anual, principalmente concentrada en primavera y otoño. Los valores de temperatura promedios, máximos y mínimos son $13,7,24,0$ y $4,5^{\circ} \mathrm{C}$, respectivamente. 
Se empleó una parcela de $1500 \mathrm{~m}^{2}$ de viña adulta (Vitis vinífera L.) y el estudio se llevó a cabo sobre la cv. Airén, con un marco de plantación de 1,5 m entre plantas, y de $3 \mathrm{~m}$ entre hileras, con una orientación $165^{\circ}$ NO-SE. Durante todo el experimento, el cultivo se mantuvo sin restricciones hídricas mediante un sistema de riego por goteo.

Se instalaron 5 radiómetros Apogee SI-121 en un mástil situado en el centro de una hilera. Estos instrumentos están dotados de una banda ancha (8-14 $\mu \mathrm{m})$ y tienen una precisión de $\pm 0,2^{\circ} \mathrm{C}$ y un ángulo de visión de $18^{\circ}$. Dos de estos radiómetros apuntaban directamente a las hojas, por ambos lados de la planta, y otros dos apuntaban hacia el suelo a ambos lados de la hilera. Un quinto radiómetro, apuntando al cielo, registraba la radiación de onda larga descendente, necesaria para una adecuada corrección atmosférica de las medidas térmicas. Un sensor CNR4, situado a $4 \mathrm{~m}$ de altura, registraba la radiación neta, y gracias a un conjunto de platos de flujo HFP01SC, enterrados a $8 \mathrm{~cm}$ de profundidad, se midió el flujo de calor en el suelo. Además se realizaron medidas de temperatura del aire, humedad relativa, velocidad del viento, radiación solar, y temperatura y humedad del suelo. Todos los datos se almacenaron cada 15 minutos en un datalogger CR1000, y posteriormente se promediaron a escalas horaria y diaria. Asimismo, se midió semanalmente la proporción de vegetación $\left(P_{v}\right)$ y la altura del cultivo $(h)$.

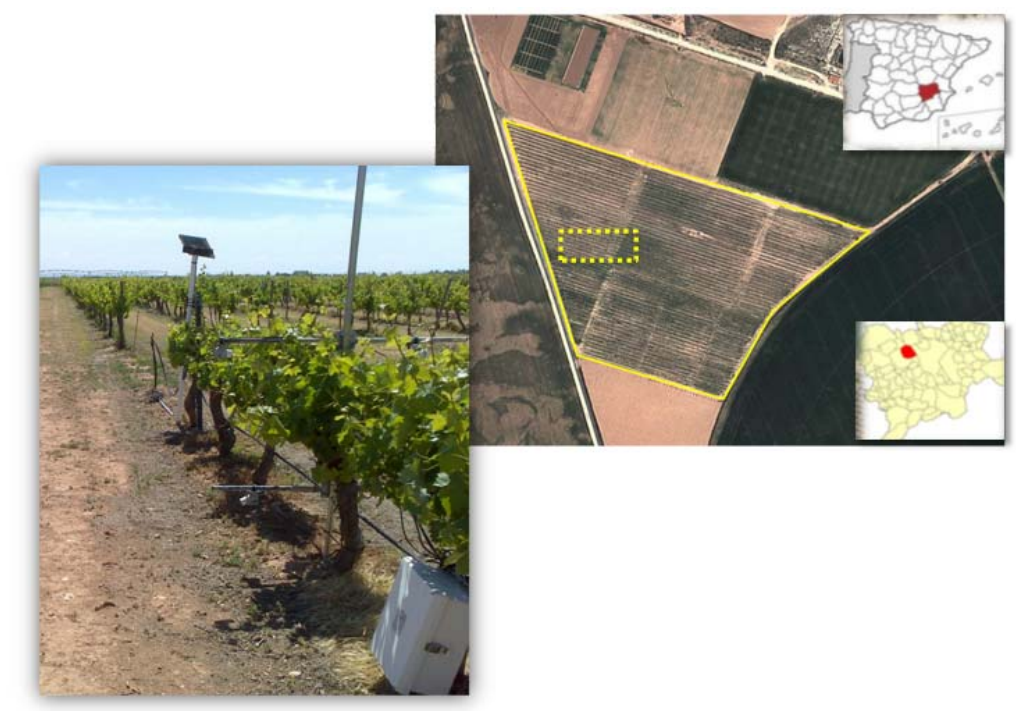

Figura 1. Localización de la zona experimental y fotografía de parte del montaje instrumental sobre la viña.

En este trabajo se aplicó el modelo simplificado de balance de energía de dos fuentes (STSEB) desarrollado por Sánchez et al. (2008) para el cálculo de la radiación neta, el flujo de calor en el suelo, el flujo de calor sensible, y la evapotranspiración. El esquema del STSEB permite, además, establecer un balance de energía por separado entre las componentes de suelo y de vegetación que conduce a una distinción entre evaporación y transpiración.

\section{3- Resultados y discusión}

Una vez corregidas de emisividad y efectos atmosféricos (Sánchez et al. 2008, 2014) los valores de temperatura de la planta $\left(T_{c}\right)$ y temperatura del suelo $\left(T_{s}\right)$, se combinaron con las variables meteorológicas y los parámetros biofísicos para obtener resultados de radiación neta $\left(R_{n}\right)$, flujo de calor en el suelo $(G)$, flujo de calor sensible $(H)$, y flujo de calor latente (LE), cada 15 minutos. Posteriormente los resultados se agregaron a escala horaria y diaria para el total de los 130 días correspondiente al periodo de estudio comprendido entre los días julianos 173 y 303, abarcando desde la floración hasta la senescencia. La figura 2a 
muestra un ejemplo de evolución diurna de las cuatro componentes de temperatura medidas por los radiómetros instalados. Las medidas de temperatura de suelo y vegetación presentan diferencias importantes a ambos lados de la hilera, mostrando la necesidad de una adecuada caracterización térmica de la viña. A modo de ejemplo, y para la misma fecha, en la figura $2 \mathrm{~b}$ se presenta la evolución diurna de los distintos flujos energéticos en superficie.

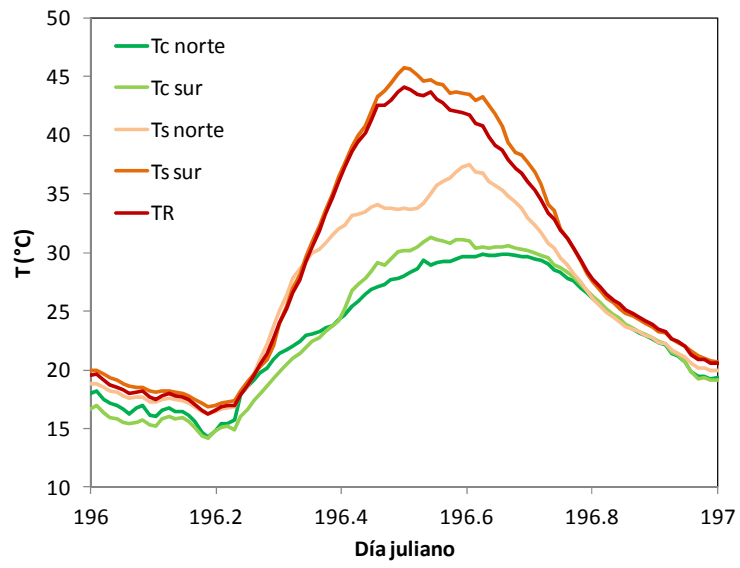

(a)

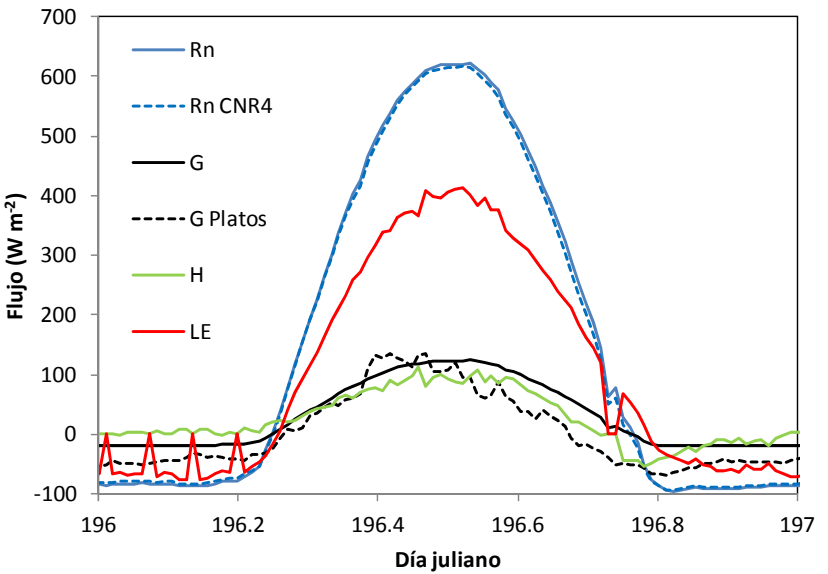

(b)

Figura 2. Ejemplo, correspondiente al día 196, de: (a) Evolución diurna de las cuatro componentes de temperatura ( $T_{c \text { norte, }}, T_{c \text { sur, }}, T_{s}$ norte, $T_{s \text { sur }}$, además de la temperatura del conjunto $\left(T_{R}\right)$, (b) Evolución diurna de los cuatro términos de la ecuación de balance de energía $\left(R_{n}, G, H\right.$ y $\left.L E\right)$, junto con las medidas in situ de $R_{n}$ y $G$.

Se observa un buen acuerdo entre los valores medidos y estimados de radiación neta (Figura 3), con un ajuste lineal muy próximo a la línea $1: 1$, y con un error cuadrático medio inferior a los $\pm 30 \mathrm{~W} \mathrm{~m}^{-2}$. La comparación entre los valores medidos y estimados de

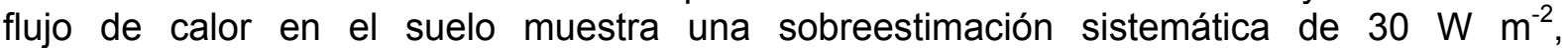
principalmente por la noche.
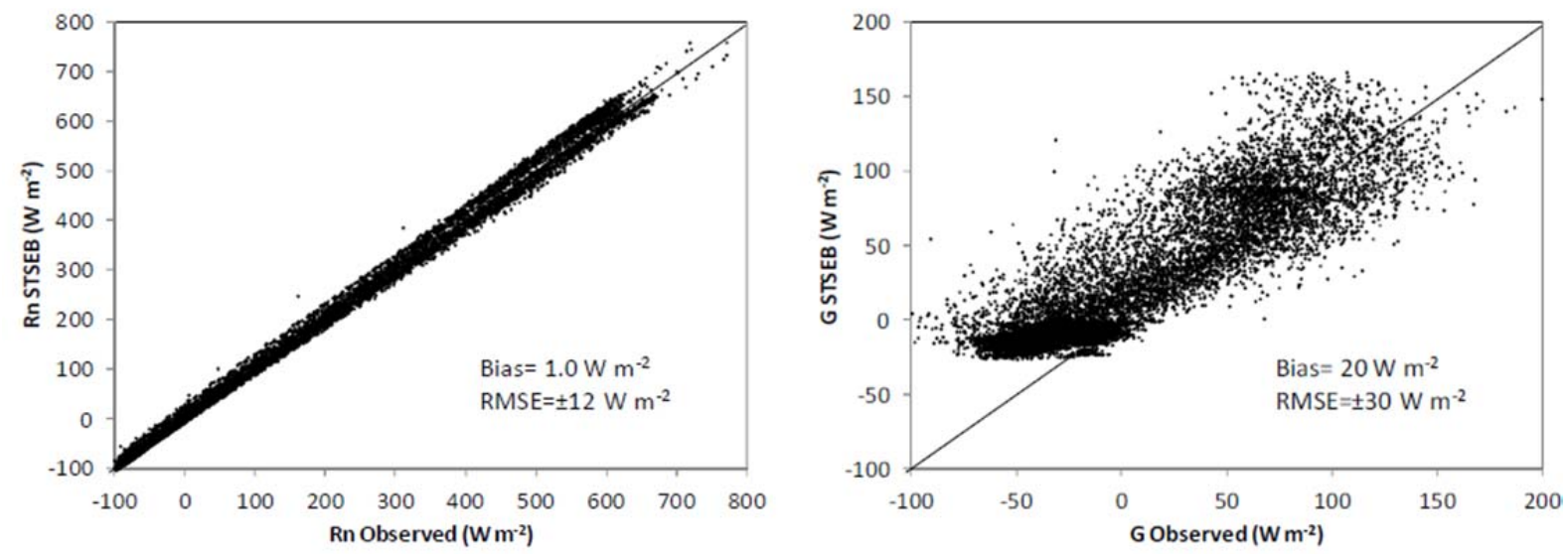

Figura 3. Comparación entre los valores medidos y estimados de radiación neta, $R_{n}$ (izquierda) y de flujo de calor en el suelo, G (derecha).

A escala diaria, la Tabla 1 muestra los valores promedio de $\mathrm{ET}_{\mathrm{c}}$ por meses entre julio y octubre. El valor máximo se alcanza entre julio y agosto, con una $E T_{c}$ de $3,9 \mathrm{~mm} \mathrm{~d}^{-1}$, 
debido a que hay una mayor demanda evaporativa y el cultivo llega al valor máximo de proporción de vegetación, descendiendo a partir de entonces.

Tabla 1. Promedio de los valores diarios de evapotranspiración de cultivo $\left(E T_{c}\right)$, y de su partición en evaporación (E) y transpiración $(T)$.

\begin{tabular}{ccccc}
\hline & Julio & Agosto & Septiembre & Octubre \\
\cline { 2 - 5 } $\mathbf{E T}_{\mathrm{c}}(\mathbf{m m})$ & 3.9 & 3.9 & 3.3 & 1.8 \\
$\mathbf{T}(\mathbf{m m})$ & $2.9(74)^{*}$ & $3.1(79)$ & $2.3(70)$ & $0.9(50)$ \\
$\mathbf{E}(\mathbf{m m})$ & $1.0(26)$ & $0.8(21)$ & $1.0(30)$ & $0.9(50)$ \\
\hline
\end{tabular}

Aplicando el balance de energía por separado al suelo y a la vegetación se obtienen los resultados de evaporación y transpiración. Los valores diarios de $\mathrm{E}$ se sitúan en torno a $1,0 \mathrm{~mm} \mathrm{~d}^{-1}$, y son bastante estables para toda la temporada, mientras que los valores diarios de $\mathrm{T}$ oscilan entre 0,9 y $3,1 \mathrm{~mm} \mathrm{~d}^{-1}$, alcanzando el máximo en agosto. En porcentaje, la transpiración supone más de un $70 \%$ del total de $\mathrm{ET}_{\mathrm{c}}$ entre julio y septiembre, llegando a alcanzar casi el $80 \%$ en agosto, coincidiendo con el estado fenológico de maduración de la uva. En términos de valores acumulados para toda la duración del experimento (130 días), del total de los $410 \mathrm{~mm}$ de $\mathrm{ET}_{\mathrm{c}}$ estimados, $290 \mathrm{~mm}$ correspondieron a transpiración de las plantas, y el resto $(120 \mathrm{~mm}$ ) a evaporación (Figura 4). Esto supone que un $30 \%$ del total de $\mathrm{ET}_{\mathrm{c}}$ en la viña corresponde a evaporación del agua del suelo. Si bien no se dispuso de medidas directas de evaporación y transpiración para validar, estos resultados dan una buena idea de la separación $\mathrm{E} / \mathrm{T}$ en viña, y ponen de manifiesto el alto porcentaje de la $E T_{\mathrm{c}}$ que se pierde en evaporación.

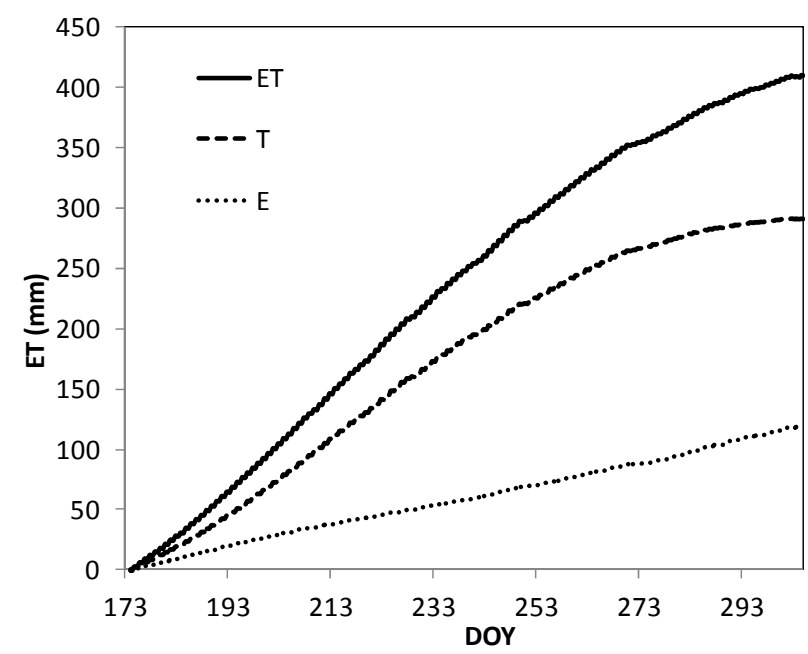

Figura 4. Evolución de los valores acumulados de evapotranspiración de cultivo (ET $)$, y de su partición en evaporación (E) y transpiración $(T)$, para toda la duración de la campaña.

\section{4- Conclusiones}

Las principales conclusiones que se pueden extraer de este trabajo son las siguientes. Las temperaturas radiométricas del suelo y de la vegetación, como parámetros de entrada en los modelos de dos fuentes, permiten obtener información de los flujos energéticos en superficie totales y realizar un balance por separado de las componentes del suelo y de la vegetación. Sin embargo, para disponer de una buena caracterización térmica en cultivos en hilera es fundamental un adecuado promediado de las partes soleadas y 
sombreadas de los distintos elementos. El valor promedio de $\mathrm{ET}_{\mathrm{c}}$ diario para el periodo de estudio resultó $3,1 \mathrm{~mm}$ día $^{-1}$, con un término de evaporación representando un $30 \%$ del total de $\mathrm{ET}_{\mathrm{c}}$ para toda la campaña. Estos resultados muestran el potencial de la caracterización térmica de la viña para una mejor programación y gestión de los riegos.

\section{Agradecimientos}

Este trabajo ha sido financiado conjuntamente por el Ministerio de Economía y Competitividad (proyectos CGL2013-46862-C2-1/2-P, cofinanciado con Fondos FEDER de la Unión Europea, y AGL2014-54201-C4-4-R), el Instituto Nacional de Investigaciones Agrarias (INIA) (proyectos RTA 2011-00100-C05-04 y RTA 2014-00049-C05-03), y la Generalitat Valenciana (proyecto PROMETEOII/2014/086).

\section{Bibliografía}

Campos, I., Neale, C.M.U., Calera, A., Balbontin, C., and González-Piqueras, J. (2010). Assessing satellite-based basal crop coefficients for irrigated grapes. Agri. Water Mgt.. 49, 211-224.

Colaizzi, P.D., Kustas, W.P., Anderson, M.C., Agam, N., Tolk, J.A., Evett, S.R., Howell, T.A., Gowda, P.H., and 'Shaughnessy, S.A. (2012). Two-source energy balance model estimates of evapotranspiration using component and composite surface temperatures. Adv. Water Res., 50, 134-151.

Evett, S.R., Kustas, W.P., Gowda, P.H., Anderson, M.C., Prueger, J.H., and Howell, T.A. (2012). Overview of the Bushland Evapotranspiration and Agricultural Remote Sensing Experiment 2008 (BEAREX08): A field experiment evaluating methods for quantifying ET at multiple scales. Adv. Water Res., 50, 4-19.

González-Dugo, M.P., González-Piqueras, J., Campos, I., Balbontin, C., and Calera, A. (2010). Estimation of surface energy fluxes in vineyard using meausurements of canopy and soil temperature. Proc. Remote Sensing and Hydrology Symposium, 27-30 September 2010, Jackson Hole, USA.

Ham, J.M., Heilman, J.L., and Lascano, R.J., (1990). Determination of soil water evaporation and transpiration from energy balance and stem flow measurements, Agri. Forest Meteorology, 52, 287-301.

Kustas, W.P., Alfieri, J.G., Anderson, M.C., Colaizzi, P.D., Prueger, J.H., Evett, S.R., Neale, C.M.U., French, A.N., Hipps, L.E., Chavez, J.L., Copeland, K.S., and Howell, T.A. (2012). Evaluating the two-source energy balance model using local thermal and surface flux observations in a strongly advective irrigated agricultural area. Adv. Water Res., 50, 120-133

Miglietta, F., Gioli, B., Brunet, Y., Hutjes, R.W.W., Matese, A., Sarrat, C., and Zaldei, A. (2009). Sensible and latent heat flux from radiometric surface at the regional scale: methodology and evaluation. Biogeosciences, 6, 1975-1986.

Ortega-Farias, S.O., Poblete-Echeverria, C., and Brisson, N. (2010). Parameterization of a two-layer model for estimating vineyard evapotranspiration using meteorological measurements, Agri. Forest Meterology, 150, 276-286.

Sánchez, J. M., Kustas, W. P., Caselles, V., and Anderson, M., (2008). Modelling surface energy fluxes over maize using a two-source patch model and radiometric soil and canopy temperature observations. Remote Sens. Environ., 112, 1130-1143.

Sánchez, J.M., López-Urrea, R., Rubio, E., and Caselles, V. (2011). Determining water use of sorghum from two-source energy balance and radiometric temperatures. Hydrol. Earth Syst. Sc., 15, 3061-3070.

Sánchez, J.M., Lopez-Urrea, R., Rubio, E., Gonzalez-Piqueras, J., and Caselles, V. (2014). Assessing crop coefficients of sunflower and canola using two-source energy balance and thermal radiometry. Agri. Water Mgt., 137, 23-29.

Sánchez, J.M., de la Cruz, F., Martinez, L., Montoro, A., and Lopez-Urrea, R. (2015). Testing the potential of two source surface energy balance to monitor plant transpiration in 
vineyards. Proc. 29th International Horticultural Congress, 17-22 August 2014, Queensland, Australia.

Shapland, T.M., Snyder, R.L., Smart, D.R., and Williams, L.E. (2012). Estimation of actual evapotranspiration in winegrape vineyards located on hillside terrain using surface renewal analysis. Irrig. Sci., 30 (6), 471-484.

Wilson, K.B., Hanson, P.J., Mulholland, P.J., Baldocchi, D.D., and Wullschleger, S.D. (2001). A comparison of methods for determining forest evapotranspiration and its components: sap-flow, soil water budget, eddy covariance and catchment water balance. Agri. Forest Meteorology., 106, 153-168.

Yunusa, I.A.M., Walker, R.R., and Lu, P., (2004). Evapotranspiration components from energy balance, sapflow and microlysimetry techniques for an irrigated vineyard in inland Australia. Agri. Forest Meteorology., 127, 93-107.

Zhang, B., Kang, S., Li, F., Tong, L., and Du, T. (2010). Variation in vineyard evapotranspiration in an arid region of northwest China. Agr.Water Mgt.., 97, 18981904. 